\title{
Haemophilus influenzae Lipopolysaccharide-induced Blood Brain Barrier Permeability during Experimental Meningitis in the Rat
}

\author{
Brian Wispelwey, * Alan J. Lesse,“ Eric J. Hansen," and W. Michael Scheld*5 \\ Departments of ${ }^{*}$ Internal Medicine and ${ }^{\S}$ Neurosurgery, University of Virginia School of Medicine, Charlottesville, Virginia 22908; \\ and the ${ }^{\ddagger}$ Department of Microbiology, University of Texas Health Science Center, Dallas, Texas 75235
}

\begin{abstract}
The factors responsible for blood-brain barrier (BBB) injury during bacterial meningitis are incompletely defined. We evaluated the role of Haemophilus influenzae type b (Hib) lipopolysaccharide (LPS) in the alteration of blood-brain barrier permeability (BBBP) in an adult, normal and leukopenic, rat model of meningitis. Intracisternal inoculation of Hib LPS resulted in (a) dose-dependent increases in BBBP from $2 \mathrm{pg}$ to 20 ng, with significant attenuation in the peak response after challenge with $500 \mathrm{ng}$ and $1 \mu \mathrm{g}$; (b) time-dependent increases in BBBP, with a delayed onset of at least $2 \mathrm{~h}$, maximum alteration at $4 \mathrm{~h}$, and complete reversal at $18 \mathrm{~h}$; (c) greater BBBP than after challenge with the live parent strain; $(d)$ and a close correlation $(r=0.86)$ between CSF pleocytosis and BBBP at 4 h. The LPS effect was significantly inhibited by preincubation with Polymyxin B and neutrophil acyloxyacyl hydrolase, however two different oligoșaccharide-specific monoclonal antibodies did not inhibit activity. No change in BBBP after inoculation with Hib LPS occurred in leukopenic rats. Hib LPS, in the setting of an intact leukocyte response, exerts profound effects on BBBP.
\end{abstract}

\section{Introduction}

The mortality and morbidity due to bacterial meningitis has remained largely unchanged over the last $30 \mathrm{yr}$ despite the development of potent and specific antimicrobial therapy for the most frequently implicated pathogens $(1,2)$. It is postulated that alterations in blood-brain barrier (BBB) ${ }^{1}$ function secondary to infection may result in irreversible and often progressive brain damage despite bacteriologic cure (3). The

Presented in part at the Annual Meeting of the Southern Society for Clinical Investigation in New Orleans, LA, January 1987 and published in abstract form (1987. Clin Res. 35: 42a).

Address reprint requests to Dr. Scheld, Division of Infectious Diseases, Box 385, University of Virginia School of Medicine, Charlottesville, VA 22908.

Received for publication 8 October 1987 and in revised form 16 May 1988.

1. Abbreviations used in this paper: $\mathrm{BBB}$, blood brain barrier, $\mathrm{BBBP}$, blood brain barrier permeability; $\mathrm{CP}$, cyclophosphamide; CSF, cerebrospinal fluid, DL42, a clinical isolate of $H$. influenzae type b; Hib, Haemophilus influenzae type b; LOS, lipooligosaccharide; PMB, Polymyxin B; PWBC, peripheral white blood cell concentration(s); WBC, white blood cell concentration(s).

J. Clin. Invest.

(C) The American Society for Clinical Investigation, Inc.

0021-9738/88/10/1339/08 \$2.00

Volume 82, October 1988, 1339-1346 factors, either host or bacterial, responsible for this damage are incompletely defined, and further understanding of these factors is essential if significant improvements in the prognosis of this disease are to be realized.

Haemophilus influenzae type $\mathrm{b}$ (Hib) remains the most common bacterial etiology of meningitis (2). The polysaccharide capsule of this organism is clearly a virulence factor for the development of invasive disease, including meningitis (4); however, there is little evidence that it has a direct role in inducing changes in blood-brain barrier permeability (BBBP) or inflammation within the cerebrospinal fluid (CSF) (5). In previous work from this laboratory an experimental model of meningitis in an adult rat was developed and characterized (6), and it was observed that encapsulation of $\mathrm{Hib}$ was not essential for BBB injury, but did facilitate its progression by allowing the organism to evade host clearance.

Bacterial LPS has been studied extensively in both in vitro and in vivo experimental models and exhibits a vast array of biological effects (7). LPS has been observed to damage vascular endothelium by either direct or indirect means (8-14) and therefore may be important in the pathogenesis and pathophysiology of BBB damage during meningitis. Only a few studies have been done previously to specifically address this question. A study from 1958 (15) evaluated BBB integrity as demonstrated by the barrier's ability to exclude vital dyes. After intracarotid injection of Escherichia coli LPS into a rabbit, reproducible diffuse breakdown in the BBB occurred. The changes in permeability were greatest if the dyes were given between 3 and $6 \mathrm{~h}$ after LPS injection. In a second study (16), intracarotid LPS injections in rabbits was followed in $4 \mathrm{~h}$ by injection of colloidal iron. The animals were then sacrificed and the brains studied by electron microscopy. In the LPStreated animals, iron was seen in large quantity within the endothelial cells, basement membrane and glial processes; however, in the saline controls, the iron remained confined to the vascular lumen. In a third study (17), microscopic evaluation of brains from cats that had received intracisternal injections of $E$. coli LPS revealed evidence of profound inflammatory cell infiltration and microcirculatory impairment in both meningeal and cortical microvessels. A potential problem with these studies is that each used extremely high doses of LPS $(\sim 50 \mu \mathrm{g})$ or systemic administration only and therefore the observed effects may not be representative of what occurs during clinical meningitis. The LPS of Hib has only recently been studied and characterized in more detail. It differs from the classic enteric LPS molecule in that it lacks any detectable O-antigen repeat unit and therefore consists of a lipid A moiety covalently coupled to a structure equivalent to the core oligosaccharide of enteric LPS molecules (18). It is sometimes referred to as a lipooligosaccharide (LOS) (19). Recently, intracisternal inoculation of Hib LPS into rabbits was observed to be a potent inducer of CSF inflammation (5). This inflam- 
mation was dose dependent and peaked between 3 and $6 \mathrm{~h}$ after inoculation. However, a significant attenuation in the LPS-induced inflammatory response could not be achieved by preincubation of LPS with either polymixin B or neutrophil acyloxyacyl hydrolase leaving in doubt the presumed predominant role for the lipid A moiety in inducing this inflammation. Additionally, a recent study (20) evaluating the role of leukocytes in the brain edema response during Streptococcus pneumoniae meningitis came to the conclusion that leukocytes $(\mathrm{PMN})$ were without effect on brain edema $24 \mathrm{~h}$ into infection, raising significant questions concerning the importance of the observation of CSF pleocytosis following Hib LPS challenge without concomitant evaluation of a physiologic variable such as brain edema response or BBBP.

The purpose of the current study therefore, was to evaluate the effect of intracisternal inoculation of isolated Hib LPS on BBBP in an adult rat model and to compare this with the effect induced following inoculation with the live parent organism. Both normal and leukopenic rats were utilized to better define the role of the host leukocyte in the induction of BBB injury during meningitis.

\section{Methods}

Challenge inocula. Hib strain DL42, a clinical isolate from an invasive infection, was provided by Dr. E. Hansen (Dallas, TX). It was grown in brain heart infusion broth (Gibco Laboratories, Madison, WI) with 5\% Fildes supplement (Difco Laboratories, Detroit, MI) to late log phase, then frozen in skim milk at $-70^{\circ} \mathrm{C}$ until use. For each experiment, an overnight growth was subcultured and allowed to grow for $8 \mathrm{~h}$ (in late $\log$ phase), then centrifuged at $2,500 \mathrm{~g}$ for $15 \mathrm{~min}$ at $20^{\circ} \mathrm{C}$, washed twice, and resuspended in saline to an approximate concentration of $10^{8} \mathrm{CFU} / \mathrm{ml}$ as determined by plating 100 -fold serial dilutions of the organisms on chocolate agar (BBL, Cockeysville, MD). The final inoculum was $10^{6-6.5} \mathrm{CFU}$ per rat.

The isolated LPS was also provided by Dr. E. Hansen and was purified from an unencapsulated variant of DL42 by the hot phenol water method of Westphal (21), as modified by Johnson and Perry (22). This purified LPS was resolved by SDS-PAGE and stained with silver to detect carbohydrates and with Coomassie Blue to detect proteins. Silver staining revealed a single LPS band migrating at approximately the same position as the LPS of a Rb LPS mutant of Salmonella typhimurium. Coomassie Blue staining showed no detectable protein in the LPS preparation. It represents LPS antigenic group 2 as defined by a set of two monoclonal antibodies to the oligosaccharide region previously described by Gulig et al. (23). Group 2 represented the most common LPS type (62\%) present in a group of 126 Hib strains isolated from invasive infections (23). Various doses of LPS were used (see below) with $20 \mathrm{ng}$ calculated to be equivalent to the amount of LPS present in $2 \times 10^{6}$ whole organisms (24), based on the following assumptions. This calculation is based on the fact that an average $E$. coli $B / r$ cell has an LPS molecule with a molecular weight of 4,300 , which is very similar to that of Hib LPS (24). The percentage of total dry weight of the cell that is LPS is 3.4 (24). The dry weight of the average gram negative bacterial cell is $2.8 \times 10^{-13} \mathrm{~g}$, such that each cell contains 9.52 $\times 10^{-15} \mathrm{~g}$ LPS (24). Therefore, $10^{5}$ Hib cells contain $1 \mathrm{ng}$ of LPS in their outer membranes. Each sample was sonicated in a jewelry sonicator (Standard Products, Whitman, MA) before inoculation.

The DL42 LPS specific murine monoclonal antibodies 4C4 and $5 G 8$ were provided by Dr. E. Hansen. These IgG antibodies have been characterized as being directed against cell surface exposed LPS antigenic determinants on the oligosaccharide portion of the LPS (23). $6 \mu \mathrm{g}$ of either antibody was incubated with $20 \mathrm{ng}$ LPS in $0.1 \%$ low pyrogen BSA (Sigma Chemical Co., St. Louis, MO) for $60 \mathrm{~min}$ at $37^{\circ} \mathrm{C}$ before inoculation. $1 \mu \mathrm{g}$ of antibody is calculated to bind at least $20 \mathrm{ng}$ LPS, assuming the molecular weight of the LPS to be $\sim 4,000$ and that of the IgG antibody to be 150,000 (18).

DL42 LPS pretreated with neutrophil acyloxyacyl hydrolase and radiolabeled but untreated DL42 LPS was provided by Robert Munford and Alice Erwin. A 20-ng inoculum of each, sonicated at $37^{\circ} \mathrm{C}$ was utilized and compared.

Polymyxin B (PMB) $(2 \mu \mathrm{g})$ (Pfizer Inc., New York) was incubated with $20 \mathrm{ng}$ of LPS for $15 \mathrm{~min}$ at $37^{\circ} \mathrm{C}$ before inoculation.

Pyrogen-free saline (Lyphomed, Inc., Rosemont, IL) was used in all control inoculations.

Induction of leukopenia. Leukopenia was induced by intraperitoneal injection of $100 \mathrm{mg} / \mathrm{kg}$ cyclophosphamide (Adria Laboratories, Columbus, $\mathrm{OH}$ ) into rats. Rats were studied $4 \mathrm{~h}, 24 \mathrm{~h}, 4 \mathrm{~d}$, and $10 \mathrm{~d}$ after this treatment. Leukopenia, defined as a white blood cell count (WBC) $<1,000 / \mathrm{mm}^{3}$ occurred reproducibly $4 \mathrm{~d}$ after cyclophosphamide treatment.

Experimental method. We used the adult rat model of meningitis previously described (6). Normal or leukopenic (100-125 g) Wistar rats (Hilltop Farms, Scottsdale, PA) were anesthetized with ketamine (Park-Davis Co., Morris Plains, NJ) $100 \mathrm{mg} / \mathrm{kg}$ and xylazine (Miles Laboratories, Swanee, KS) $7 \mathrm{mg} / \mathrm{kg}$ i.m. Cyclophosphamide-treated rats had an evaluation of peripheral WBC with a hemocytometer at the start of the experiment to ensure leukopenia (WBC $<1,000$ cells/ $\mathrm{mm}^{3}$ ). The rats were then inoculated intracisternally with a 25-gauge butterfly needle fitted to a micromanipulator. After removal of 50-75 $\mu \mathrm{l}$ of CSF, $50 \mu \mathrm{l}$ of a given inoculum (described above) was injected directly into the cisterna magna. Only inoculations without visible blood contamination were evaluated. The rats were then allowed to progress for varying time intervals (as outlined in Results). At a given evaluation time point, the rat was again anesthetized and the CSF sampled by the above described method. $25 \mu$ micropipette (Dade Scientific Corp., Miami, FL) samples were collected for quantitative culture, CSF WBC and evaluation of BBBP (see below). Peripheral blood was collected at simultaneous time points for quantitative culture and evaluation of BBBP (see below).

Assessment of BBBP. $1 \mathrm{~h}$ before each evaluation time point, each rat was given $\approx 10 \mu \mathrm{Ci}$ of ${ }^{125}$ I-labeled BSA (ICN Pharmaceuticals, Irvine, $\mathrm{CA}$ ) via intracardiac injection. At a given evaluation time point, $25 \mu \mathrm{l}$ of both CSF and blood were obtained and placed in individual 1 dram vials and the samples were counted in a gamma 300 counter (Beckman Instruments, Inc., Irvine, CA) for $10 \mathrm{~min}$. The permeability of the blood-brain barrier was then calculated as the counts per minute $\mathrm{CSF} /$ counts per minute blood $\times 100$ and expressed as a percentage. This method has previously been shown to correlate positively with the degree of morphologic change in the BBB during experimental meningitis (6).

Analysis. Statistical comparisons between groups were done by Student's $t$ test and $P$ values $\leq 0.05$ were considered significant.

\section{Results}

Inoculation with DL42 LPS vs. live DL42. DL42 LPS (20 ng) and live DL42 $\left(10^{6.5} \mathrm{CFU}\right)$ were compared with regard to their effects on BBBP and CSF WBC 4 and $18 \mathrm{~h}$ after intracisternal inoculation. The results are depicted in Table I. $20 \mathrm{ng}$ of LPS resulted in a marked CSF pleocytosis with $\sim 20,000 \mathrm{WBC}$ present at both 4 and $18 \mathrm{~h}$. The live DL42 inoculum, despite CSF colony counts of $\sim 10^{7} / \mathrm{ml}$ at both 4 and $18 \mathrm{~h}$, had significantly lower $(P<0.05)$ CSF WBC at 4 and $18 \mathrm{~h}$, respectively. The BBBP after LPS inoculation was $7.97 \%$ at $4 \mathrm{~h}$, which was significantly greater $(P<0.02)$ than the value $(1.59 \%)$ observed following live DL42 inoculation. Both values were significantly greater $(P<0.01)$ than the 4 -h control value of $0.37 \%$. At $18 \mathrm{~h}$, however, the BBBP following LPS inoculation had decreased to $0.41 \%$, which was not significantly greater than controls. In the live DL42 inoculated rats, the 18-h BBBP 


\begin{tabular}{|c|c|c|c|c|}
\hline \multirow[b]{2}{*}{ Inoculum } & \multicolumn{4}{|c|}{ Time after inoculation } \\
\hline & CSF WBC* & BBBP $^{\ddagger}$ & CSF WBC* & BBBP \\
\hline & \multicolumn{2}{|c|}{$4 h($ mean $\pm S E)$} & \multicolumn{2}{|c|}{$18 h($ mean $\pm S E)$} \\
\hline \multirow[t]{2}{*}{ LPS 20 ng } & $24,294 \pm 3,252^{\S}$ & $7.97 \pm 0.99^{\S}$ & $19,900 \pm 4,682^{\S}$ & $0.41 \pm 0.04$ \\
\hline & \multicolumn{2}{|c|}{$(n=17)$} & \multicolumn{2}{|c|}{$(n=4)$} \\
\hline $10^{6.5}$ CFU Hib & $1,262 \pm 688$ & $1.59 \pm 0.31$ & $8,020 \pm 2,389$ & $0.99 \pm 0.11^{1}$ \\
\hline Saline & $0 \pm 0$ & $0.37 \pm 0.05$ & $10 \pm 10$ & $0.18 \pm 0.13$ \\
\hline
\end{tabular}

* Leukocytes $/ \mathrm{mm}^{3}$. ${ }^{\ddagger} \mathrm{cpm} \mathrm{CSF} / \mathrm{cpm}$ blood $\times 100$, expressed as \%. ${ }^{\S} P \leq 0.05$ compared to Hib and controls. "Log $10 \mathrm{CSF}$ CFU/ml $=7.21 \pm 0.20($ mean \pm SE $)(4 \mathrm{~h})$ and $7.28 \pm 0.15($ mean \pm SE $)(18 \mathrm{~h}) . \quad{ }^{\prime} P \leq 0.05$ compared to LPS and controls.

of $0.99 \%$ was significantly greater $(P<0.01)$ than both LPS and controls. All of the live DL42 inoculated rats were bacteremic at both 4 and $18 \mathrm{~h}$, with blood bacterial concentrations (mean \pm SE) of $\log _{10} 5.70 \pm 0.13 \mathrm{CFU} / \mathrm{ml}$ and $\log _{10} 4.79 \pm 0.38$ $\mathrm{CFU} / \mathrm{ml}$ at 4 and $18 \mathrm{~h}$, respectively.

Kinetics of changes in CSF WBC and BBBP after LPS inoculation. Multiple time points were evaluated to assess the kinetics of onset and reversal in BBBP following intracisternal LPS (20 ng) inoculation (Table II, Fig. 1). $2 \mathrm{~h}$ after inoculation, both CSF WBC and BBBP were not different from salinechallenged controls. At $3 \mathrm{~h}$, however, significant $(P<0.05)$ increases in both parameters were observed. The maximal increase in BBBP was observed at $4 \mathrm{~h}$, before the peak CSF WBC at $8 \mathrm{~h}$. At 6 and $8 \mathrm{~h}$ the BBBP had decreased significantly $(P$ $<0.02$ ) from the observed peak value, returning to control levels at $18 \mathrm{~h}$, despite a persistent CSF pleocytosis.

LPS dose response. Inoculation of multiple doses of LPS were evaluated at the 4-h time point (the point of maximal

Table II. CSF WBC and BBBP at Varying Times after LPS (20 ng) Inoculation

\begin{tabular}{|c|c|c|c|c|}
\hline \multirow[b]{2}{*}{ Hours } & \multicolumn{2}{|c|}{ CSF WBC } & \multicolumn{2}{|c|}{ BBBP $^{\ddagger}$} \\
\hline & LPS & Control & LPS & Control \\
\hline & \multicolumn{2}{|c|}{$M e a n \pm S E$} & \multicolumn{2}{|c|}{$M e a n \pm S E$} \\
\hline 2 & $\begin{array}{c}0 \pm 0 \\
(n=5)\end{array}$ & $\begin{array}{c}0 \pm 0 \\
(n=3)\end{array}$ & $0.15 \pm 0.04$ & $0.08 \pm 0.04$ \\
\hline 3 & $\begin{array}{c}1,030 \pm 345^{\S} \\
(n=4)\end{array}$ & $\begin{array}{c}0 \pm 0 \\
(n=3)\end{array}$ & $1.03 \pm 0.25^{\S}$ & $0.11 \pm 0.01$ \\
\hline 4 & $\begin{array}{c}24,294 \pm 3,252^{8} \\
(n=17)\end{array}$ & $\begin{array}{c}0 \pm 0 \\
(n=9)\end{array}$ & $7.97 \pm 0.99^{\S ॥}$ & $0.37 \pm 0.05$ \\
\hline 6 & $\begin{array}{c}36,393 \pm 3,834^{\S} \\
(n=7)\end{array}$ & $\begin{array}{c}0 \pm 0 \\
(n=3)\end{array}$ & $3.43 \pm 0.55^{\S}$ & $0.35 \pm 0.02$ \\
\hline 8 & $\begin{array}{c}42,556 \pm 6,014^{\delta} \\
(n=8)\end{array}$ & $\begin{array}{c}0 \pm 0 \\
(n=5)\end{array}$ & $1.22 \pm 0.11^{\S}$ & $0.35 \pm 0.06$ \\
\hline 18 & $\begin{array}{c}19,900 \pm 4,682^{\S} \\
(n=4)\end{array}$ & $\begin{array}{l}10 \pm 10 \\
(n=5)\end{array}$ & $0.41 \pm 0.04$ & $0.18 \pm 0.13$ \\
\hline
\end{tabular}

* Leukocytes $/ \mathrm{mm}^{3}$.

${ }^{\ddagger} \mathrm{cpm} \mathrm{CSF} / \mathrm{cpm}$ blood, expressed as \%.

${ }^{8} P<0.05$ compared to control.

" $P<0.05$ compared to $2,3,6,8$ and $18 \mathrm{~h}$.
BBBP with $20 \mathrm{ng}$ LPS). As noted in Table III, 2 pg represented the threshold dose in our model, as only $1 / 7$ rats showed any activity, with the overall mean values for CSF WBC and BBBP not significantly different than controls. At $200 \mathrm{pg}$, the response was more dramatic; however, due to a large standard deviation in the BBBP response only the CSF WBC increase reached statistical significance. The peak response was observed at a dose of $20 \mathrm{ng}$. Inoculation with $500 \mathrm{ng}$ and $1 \mu \mathrm{g}$ resulted in a reproducible significant $(P<0.01)$ attenuation in the maximal response. A strong correlation between CSF WBC and BBBP was observed $(r=0.86, P<0.001)$ for all doses of LPS at the 4-h time point (Fig. 2).

LPS inoculation into leukopenic rats (Table IV). Rats were evaluated either $4 \mathrm{~h}, 24 \mathrm{~h}, 4 \mathrm{~d}$ or $10 \mathrm{~d}$ after cyclophosphamide (CP) treatment to better assess the role of the host PMN in the induction of BBBP following LPS inoculation and how this might relate to varying degrees of PMN depletion. Each group was inoculated intracisternally with LPS (20 ng) and BBBP and CSF pleocytosis was assessed $4 \mathrm{~h}$ later. Peripheral WBC (PWBC) was significantly decreased at $4 \mathrm{~h}, 24 \mathrm{~h}$, and $4 \mathrm{~d}$ after $\mathrm{CP}$ treatment compared with non-CP-treated rats $(P<0.001)$. The BBBP in each of these groups of $\mathrm{CP}$ treated rats was also significantly less $(P<0.05)$ than in the non-CP-treated rats. BBBP after inoculation of LPS into rats that were profoundly leukopenic ( $4 \mathrm{~d}$ post-CP) was no different than that observed in saline inoculated rats $(P=0.6)$. The peripheral WBC in rats

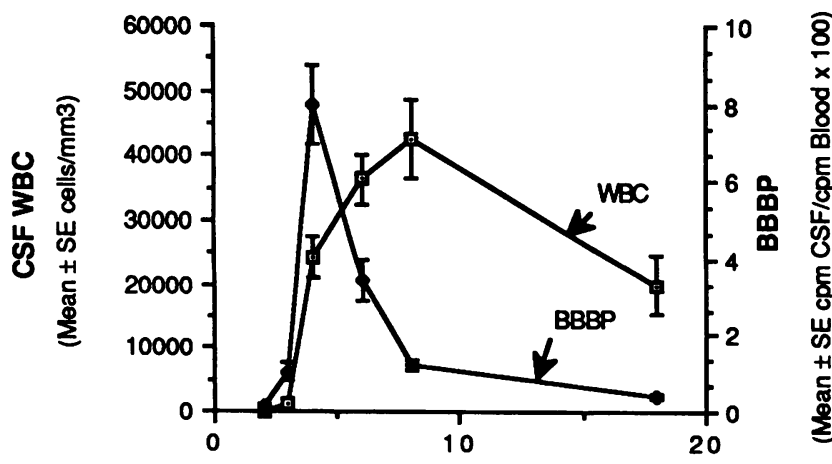

TIME (H)

Figure 1. Kinetics of changes in CSF WBC and BBBP after LPS inoculation. 
Table III. H. influenzae LPS Dose Response: Time after Inoculation $=4 \mathrm{~h}$

\begin{tabular}{ccc}
\hline Dose & CSF WBC* & BBBP* \\
\hline $2 \mathrm{pg}(n=7)$ & $479 \pm 479$ & $0.52 \pm 0.23$ \\
$200 \mathrm{pg}(n=7)$ & $9,836 \pm 4,336^{\S}$ & $2.61 \pm 1.19$ \\
$20 \mathrm{ng}(n=17)$ & $24,294 \pm 3,252^{\S}$ & $7.97 \pm 0.99^{\S}$ \\
$500 \mathrm{ng}(n=9)$ & $2,133 \pm 1,032$ & $2.37 \pm 0.65^{\S}$ \\
$1 \mu \mathrm{g}(n=5)$ & $1,580 \pm 1,144$ & $1.77 \pm 0.59^{8}$ \\
Saline $(n=9)$ & $0.0 \pm 0.0$ & $0.37 \pm 0.05$ \\
\hline
\end{tabular}

* Mean \pm SE leukocytes $/ \mathrm{mm}^{3}$.

${ }^{\ddagger}$ Mean \pm SE cpm CSF/cpm blood $\times 100$, expressed as $\%$.

$P<0.05$ compared to saline.

$10 \mathrm{~d}$ after $\mathrm{CP}$ treatment was no longer significantly different $(P$ $=0.32$ ) from that in the rats that did not receive $C P$. The BBBP in these CP treated rats, $4 \mathrm{~h}$ following LPS inoculation, was also not significantly different $(P=0.45)$ from that in the rats that did not receive $C P$.

Inhibition of LPS Effect (Tables $V$ and VI). Intracisternal inoculation of $20 \mathrm{ng}$ LPS preincubated with $2 \mu \mathrm{g}$ of PMB resulted in a significant $(P \leq 0.004)$ attenuation in the observed LPS effect on BBBP at $4 \mathrm{~h}$. PMB $(2 \mu \mathrm{g})$ inoculation alone resulted in a modest but significant $(P<0.05)$ increase in BBBP. A higher dose of PMB $(20 \mu \mathrm{g})$ produced changes in BBBP and CSF WBC as great as that seen with LPS.

The change in BBBP was also significantly less after inoculation with $20 \mathrm{ng}$ of deacylated LPS $(P<0.005)$ when compared with $20 \mathrm{ng}$ of untreated LPS at $4 \mathrm{~h}$. The control LPS ( ${ }^{3} \mathrm{H}$-labeled) used for the comparison was equivalent in activity to the LPS used in all other inoculations at the 20-ng dose $(P=0.9)$.

Preincubation of LPS with either the 4C4 or 5G8 oligosaccharide-specific monoclonal antibodies before inoculation did not result in attenuation in LPS activity at $4 \mathrm{~h}(P>0.1)$.

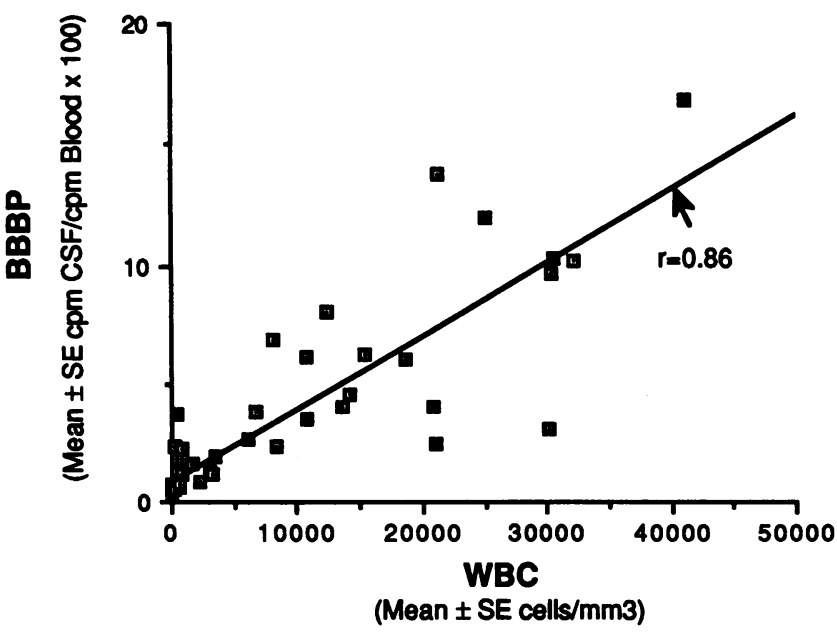

Figure 2. The relationship between CSF WBC and BBBP at 4 h postinoculation of LPS. Doses of LPS included $2 \mathrm{pg}, 200 \mathrm{pg}, 20 \mathrm{ng}, 500$ $\mathrm{ng}$, and $1 \mu \mathrm{g}$. Each point represents the values for CSF WBC and BBBP for 1 of 51 experimental animals. The line represents a best-fit simple regression analysis with $r=0.86, P<0.001$.

\section{Discussion}

Bacterial meningitis can lead to significant and often progressive abnormalities in the host's $\operatorname{BBB}(3,6)$, resulting in neurologic impairment (25-27) or death. In this study we used an adult rat model in an attempt to better define some of the factors responsible for these deleterious effects. Advantages of this model, used in two previously reported investigations (6, 28 ) by this laboratory, are that it allows direct comparison of both morphologic and physiologic or functional changes in the BBB in the same experimental animal, has the potential for in vitro culture of cerebral capillary endothelial cells with subsequent evaluation and comparison to observations in the in vivo model, and is less expensive than the previously characterized rabbit model of meningitis. A consistant criticism of this and all previous experimental models of meningitis is that it largely addresses postinductive events or pathophysiology and not pathogenesis.

Intracisternal Hib LPS inoculation into rats resulted in profound increases in BBBP and CSF WBC, which were significantly greater than those seen after inoculation with the live parent organism. This was observed despite the fact that the LPS dose $(20 \mathrm{ng}$ ) was calculated to be equivalent to the amount of LPS present in the whole organism inoculum. However, it must be emphasized that essentially nothing is known about the physical state of Hib LPS in the CSF during the course of natural infections. Hib gains access to the CSF when Hib cells invade this compartment from the bloodstream. Once present in the CSF, LPS can be released from both dying Hib cells as well as from growing cells, in the latter case in the form of blebs or outer membrane vesicles (29). The LPS in these various types of outer membrane fragments is then free to interact with CSF. Indeed, it has been suggested that the physical state of LPS (i.e., free vs. bound) may be important for its interaction with the Limulus enzyme (30) and it has been previously observed that removal of LPS from other outer membrane constituents by either hot-phenol extraction as used in this study or by naturally occurring vesicles altered the behavior of LPS (31). Our observation suggests that cell associated LPS may be relatively inactive biologically, whereas free LPS accounts for most of the observed affects. This is supported by the observations of Tauber et al. (32). They noted a significant increase in measurable LPS in the CSF following cefotaxime treatment of $E$. coli meningitis in rabbits. This increase in LPS was associated with a concomitant significant increase in brain edema compared with untreated controls. Simultaneous treatment with cefotaxime and a lipid A-directed monoclonal antibody resulted in attenuation of the brain edema response. These observations raise some practical questions with regard to antibiotic therapy of meningitis. Certain antibiotics that are efficient in causing LPS release during therapy may, at least transiently, be detrimental to the patient.

The increase in BBBP induced by the intracisternal inoculation of LPS was dose dependent. Doses in the picogram range yielded measurable abnormalities in BBBP with the peak response observed at $20 \mathrm{ng}$. This is a level of LPS readily attainable in the course of a naturally acquired infection (33). Of note, a consistent attenuation in the maximal response was observed at doses of $500 \mathrm{ng}$ and $1 \mu \mathrm{g}$. These rats were still "ill-appearing" however, despite the less prominant CSF inflammatory response. The explanation for this observation is 
Table IV. Effect of Cyclophosphamide(CP)-induced Leukopenia on BBBP $4 \mathrm{~h}$ after LPS Inoculation

\begin{tabular}{|c|c|c|c|c|}
\hline Group & Inocula & WBC & CSF WBC ${ }^{\ddagger}$ & BBBP \\
\hline 4 h p CP $(n=6)$ & LPS (20 ng) & $4,366 \pm 258^{\prime \prime}$ & $13,275 \pm 2,688$ & $3.95 \pm 0.50^{\top}$ \\
\hline 24 h p CP $(n=5)$ & LPS (20 ng) & $3,940 \pm 946^{\prime \prime}$ & $7,660 \pm 1,993$ & $2.02 \pm 0.50^{\prime}$ \\
\hline $4 \mathrm{~d} \mathrm{p} \mathrm{CP}(n=8)$ & LPS (20 ng) & $322 \pm 69^{\prime \prime}$ & $0 \pm 0$ & $0.43 \pm 0.08^{1 * *}$ \\
\hline $4 \mathrm{~d}$ p CP $(n=6)$ & Saline & $183 \pm 33^{\prime \prime}$ & $0 \pm 0$ & $0.39 \pm 0.08^{\prime}$ \\
\hline $10 \mathrm{~d} \mathrm{p} \mathrm{CP}(n=10)$ & LPS (20 ng) & $9,155 \pm 1,003$ & $18,511 \pm 3,319$ & $6.64 \pm 1.45$ \\
\hline No CP $(n=17)$ & LPS (20 ng) & \$ & $24,294 \pm 3,252$ & $7.97 \pm 0.99$ \\
\hline
\end{tabular}

* Mean \pm SE peripheral leukocytes $/ \mathrm{mm}^{3}$. ${ }^{\ddagger}$ Mean \pm SE CSF leukocytes $/ \mathrm{mm}^{3}$. ${ }^{8}$ Mean \pm SE cpm CSF/cpm blood $\times 100$. " $P<0.01 \mathrm{compared}$ to no CP. ' $P<0.05$ compared to no CP. ${ }^{* *} P>0.6$ compared to saline. ${ }^{\sharp}$ Mean WBC in no CP rats $(n=50)=10,220 \pm 426$.

uncertain, but is not without precedent. Studies comparing the effect of intraperitoneal inoculation of LPS into either LPS sensitive or resistant mice (34) revealed that LPS sensitive mice mounted a significantly increased peritoneal inflammatory response at low doses ( $7.5 \mathrm{ng})$ of LPS, but this response decreased to control levels at higher doses. LPS resistant mice remained unresponsive until high doses $(>7.5 \mu \mathrm{g})$ of LPS were inoculated, after which a progressively increased inflammatory response was observed. It is hypothesized that the LPS sensitive mice are able to respond to LPS concentrations present in the course of naturally acquired infections, but concentrations in excess of this may "overload" the immune system resulting in an antiinflammatory effect. This is consistent with the observation that LPS sensitive mice were less susceptible than LPS resistant mice to intraperitoneal infection with Salmonella typhimurium (34). An antiinflammatory effect of LPS was also noted when LPS was injected intravenously into rabbits before the induction of a reversed passive Arthus reaction (35). LPS preinjection eliminated the PMN extravasation and increased vascular permeability normally observed and PMN's taken from the LPS-treated animals were noted to be much less responsive to the chemotactic stimulus of $\mathrm{C}_{5 \mathrm{a}}$. If significant amounts of LPS enter the peripheral circulation in our model, this effect, as well as the fact that LPS has been shown to augment PMN adhesiveness and inhibit migration

Table $V$. Effect of PMB and Oligosaccharide-specific Monoclonal Antibodies on HIB LPS-induced CSF WBC and BBBP

\begin{tabular}{lcc}
\hline \multicolumn{1}{c}{ Inoculum } & CSF WBC* & \multicolumn{1}{c}{ BBBP $^{*}$} \\
\hline Saline $(n=9)$ & $0 \pm 0^{\S}$ & $0.37 \pm 0.05^{\S}$ \\
LPS $(n=17)$ & $24,294 \pm 3,252$ & $7.97 \pm 0.99$ \\
PMB $(2 \mu \mathrm{g})(n=4)$ & $25 \pm 14^{\S}$ & $0.97 \pm 0.25^{\S}$ \\
PMB $(20 \mu \mathrm{g})(n=5)$ & $17,857 \pm 6,625^{\prime \prime}$ & $6.58 \pm 1.98^{\prime \prime}$ \\
PMB $(2 \mu \mathrm{g})+$ LPS $(n=6)$ & $3,550 \pm 2,851^{\S}$ & $2.37 \pm 1.16^{\S}$ \\
4C4 $(n=4)$ & $0 \pm 0^{\S}$ & $0.35 \pm 0.14^{\S}$ \\
5G8 $(n=7)$ & $0 \pm 0^{\S}$ & $0.48 \pm 0.34^{\S}$ \\
4C4 + LPS $(n=6)$ & $27,350 \pm 9,630^{\prime \prime}$ & $11.23 \pm 4.98^{\prime \prime}$ \\
5G8 + LPS $(n=7)$ & $21,200 \pm 5,522^{\prime \prime}$ & $6.42 \pm 3.29^{\prime \prime}$ \\
\end{tabular}

\footnotetext{
* Mean \pm SE leukocytes $/ \mathrm{mm}^{3}$.

$\ddagger$ Mean \pm SE cpm CSF/cpm blood $\times 100$, expressed as $\%$.

${ }^{\&} P<0.05$ compared to LPS.

" $P>0.1$ compared to LPS.

'Oligosaccharide-specific monoclonal antibodies (see text).
}

directly (36), could potentially contribute to the decreased inflammatory response in the CSF at higher doses. An additional unproven hypothesis is that higher doses of LPS might suppress production of a required proinflammatory mediator such as IL-1 by macrophages or other cells. Consistent with this is the observation that low doses of LPS stimulate multiple alveolar macrophage functions, while higher doses lead to inhibition, exclusive of causing direct cell death $(37,38)$. Finally, a nonspecific antiinflammatory effect might result from LPSinduced release of adrenal corticosteroids, which have been hypothesized as having a significant role in modulating BBBP (39).

The increased BBBP secondary to intracisternal inoculation of Hib LPS was also time dependent. This time dependency is supported by several studies already mentioned (15-17), where peak abnormalities in BBBP were observed between 3 and $6 \mathrm{~h}$ after LPS exposure. An in vitro model evaluating PMN adhesion to cultured human endothelial cells after pretreatment with either LPS or IL-1 further supports these observations and may provide some insight with regard to the mechanism of the LPS effect. PMN adhesion to the endothelium did not increase until $>30 \mathrm{~min}$ after LPS exposure and did not reach a maximum until $4 \mathrm{~h}$ after exposure (40). This is important in light of our observation that at the point of the maximally increased BBBP in our model $(4 \mathrm{~h})$, there was a close correlation between CSF WBC and the degree of BBBP.

The role of the PMN in the induction of vascular permeability in diseases associated with the presence of LPS has been actively debated. Despite evidence that LPS can directly injure vascular endothelium (8-10), many studies suggest vascular damage is at least augmented by an intact neutrophil response or may, in fact, require it (11-14). A study evaluating the kinetics of acute inflammation in the skin of rabbits after in-

Table VI. Effect of Deacylation on HIB LPS-induced $C S F W B C$ and $B B B P$

\begin{tabular}{llc}
\hline \multicolumn{1}{c}{ Inoculum (20 ng) } & CSF WBC & BBBP \\
\hline Deacylated LPS $(n=10)$ & $13,200 \pm 6,456^{\S}$ & $3.25 \pm 1.69^{\S}$ \\
Control LPS $(n=3)$ & $24,633 \pm 2,814$ & $8.27 \pm 0.44$
\end{tabular}

\footnotetext{
* Mean \pm SE leukocytes $/ \mathrm{mm}^{3}$.

${ }^{\ddagger}$ Mean \pm SE cpm CSF/cpm blood $\times 100$, expressed as $\%$.

${ }^{\S} P<0.05$ compared to control LPS.
} 
tradermal injection of $E$. coli was recently reported (41). The time of peak PMN infiltration occurred in 3-4 h, coinciding with the time of peak vascular permeability, as noted in our study, with both effects being reversible in $\sim 8 \mathrm{~h}$. Of note, no increase in vascular permeability was noted in rabbits rendered leukopenic before $E$. coli injection, thus supporting our current experiments. In contrast, a recent study (20) found no difference in the brain edema response between normal or leukopenic rabbits $24 \mathrm{~h}$ after intracisternal inoculation of $S$. pneumoniae. However, no evaluation of these animals was performed at earlier time points to more precisely elucidate the potential role for the PMN before the onset of significant complicating variables. Our own experience with this animal model notes that the rabbits are uniformly bacteremic with high titers of bacteria at $24 \mathrm{~h}$ after intracisternal challenge $\left(\geq 10^{5} \mathrm{CFU} / \mathrm{ml}\right.$ ), are often moribund, and death before this time point is not uncommon. Therefore, in this setting it is difficult (if not impossible) to dissect out a possible role for the host leukocyte from the probable associated disseminated intravascular coagulation, local central nervous system or systemic hypoxia and hypotension or shock. All of these factors, either alone or in concert, could potentially lead to brain edema. Additionally, the brain edema response for the uninfected controls and infected normal and leukopenic rabbits exhibited a marked degree of overlap with a significant difference between uninfected controls and infected rabbits only achieved when the data for the normal and leukopenic animals were combined. In addition, brain edema during meningitis may reflect vasogenic cerebral edema (e.g., altered BBBP), and interstitial or cytotoxic edema. Therefore, any conclusion about the role of the host PMN during meningitis remains uncertain based on this report. Experimental Hib meningitis in leukopenic rats was previously observed in our laboratory to cause a significant alteration in BBBP late in the course of the disease ( $18 \mathrm{~h}$ after bacterial challenge), however this alteration was significantly less than observed in rats with an intact PMN response (28). When the same organism was evaluated $4 \mathrm{~h}$ after inoculation, BBBP was not significantly different than controls in the neutropenic animals despite observation of significantly increased BBBP in the normal animals treated similarly (unpublished data). In the current study, a direct correlation between PWBC and BBBP after LPS inoculation was observed. PWBC was significantly decreased at $4 \mathrm{~h}, 24 \mathrm{~h}$ and 4 d after CP treatment compared with non-CP-treated rats. BBBP after LPS inoculation was also significantly less in each of these groups of CP-treated rats compared with non-CPtreated rats. However, the return of PWBC to normal $10 \mathrm{~d}$ following $C P$ treatment restored the maximal increase in BBBP after LPS inoculation. Of note, BBBP after LPS inoculation into rats that were profoundly leukopenic ( $4 \mathrm{~d}$ post-CP) was no different than that observed in saline inoculated rats, further supporting the role of the host PMN in the pathophysiology of BBB damage during meningitis. The persistent CSF pleocytosis despite reversal in BBBP at later time points in normal rats is not necessarily inconsistent with this, as PMN clearance from the CSF is not immediate $(3,42)$. These PMNs may have degranulated at earlier time points and are therefore incapable of inflicting further damage. This data in no way suggests that the PMN is the only cause of increased BBBP during meningitis, however, it emphasizes that after inoculation of nonreplicating stimuli (LPS, cell wall fragments, etc.) evaluation at multiple time points is crucial before a patho- physiologic role for the PMN in BBB injury can be either affirmed or negated. This is in contrast to the situation following live organism inoculation where an ongoing stimulus for "fresh" PMN recruitment is present, resulting in ongoing, nonreversible injury.

The lipid A molecule is known to be responsible for many of the observed biological effects of LPS (7). Preincubation of Hib LPS with PMB, which binds to Lipid A and inactivates it (43), resulted in marked attenuation of the observed LPS effects. Of note, however, PMB was not without its own intrinsic activity in our model, especially when $20 \mu \mathrm{g}$ was inoculated. Recently, it was observed that PMB, in appropriate dosages, could stimulate IL-1 secretion by human monocytes and could act synergistically with LPS to induce IL-1 secretion (44). This, along with its inherent membrane toxicity at higher doses, may lead to a paradoxical increase in inflammation as was observed in our model.

Neutrophil acyloxyacyl hydrolase pretreated LPS was also significantly less active than untreated LPS. This neutrophilic enzyme selectively removes the nonhydroxylated fatty acids that are attached by acyloxyacyl linkages to the ester-and amide-linked long chain fatty acids of lipid A. It has been previously shown that this enzymatic treatment of Salmonella typhimurium LPS reduced its activity in the dermal Shwartzman reaction by a factor of $>100$ (45). More recently, it was observed that pretreatment of endothelial cells with deacylated LPS did not result in increased neutrophil adherence to these cells. Additionally, deacylated LPS inhibited LPS augmentation of neutrophil adherence to endothelial cells (46). Therefore, in vivo deacylation of LPS by the PMN may produce a competitive inhibitor for intact LPS.

The polysaccharide component of LPS was, until relatively recently, considered to be inactive biologically. Recent evidence suggests that it can initiate activation of the alternate pathway of complement (47) as well as specifically stimulate monocytes to release effector monokines (48). However, the lack of attenuation in the LPS effect by two different oligosaccharide-specific monoclonal antibodies further supports the importance of the lipid A portion of the molecule in our model.

In summary, Hib LPS clearly exerts profound effects on BBBP and CSF inflammation following intracisternal inoculation. These effects are both dose and time dependent, inhibited by agents which specifically interfere with lipid $A$ and depend on an intact neutrophil response. However, significant questions remain as to the mechanism of the LPS induced effects. In vitro, LPS is not directly chemotactic for neutrophils (49) despite the profound neutrophil accumulation noted in vivo. Therefore, it is argued that at least some of the LPS-induced effects requires the production of an endogenous substance(s) which in turn leads to PMN accumulation and subsequent vascular damage (14). LPS challenge results in the release of many potential mediators in vivo (47) and two, IL-1 and tumor necrosis factor, may prove to be particularly important. Both are endogenously produced in significant amounts following LPS challenge $(50,51)$; result in augmentation of PMN adherence to endothelial cells $(39,51)$; and IL-1 has been shown to mediate PMN migration in vitro (52). Additionally, intradermal IL-1 injection into rabbits results in peak PMN accumulation 30-60 min before the peak observed after LPS injection (52), further suggesting a role for IL-1 in mediating or potentiating the effects of LPS. Therefore, the pathogenesis 
and pathophysiology of BBB dam̆age during meningitis may represent the complex interaction of bacterial factors (e.g., LPS), endogenously produced substances and the host's neutrophil response. Further evaluation of each of these factors is necessary before rational adjunctive therapies for meningitis can be devised.

\section{Acknowledgements}

Supported in part by research grants from the National Institutes of Allergy and Infectious Diseases (AI 17904) and from the Kate Miller Jeffress and Thomas Jeffress Memorial Trust. Dr. Scheld is an Established Investigator of the American Heart Association. Dr. Wispelwey and Dr. Lesse are supported by a National Institutes of Health training grant (T 32-AI 07046).

\section{References}

1. Swartz, M. N., and P. R. Dodge. 1965. Bacterial meningitis-a review of selected aspects. N. Engl. J. Med. 272:725-731.

2. Schlech, W. F., J. I. Ward, J. D. Band, A. Hightower, D. W. Fraser, and C. V. Broome. 1985. Bacterial meningitis in the United States, 1978 through 1981 . The national bacterial meningitis surveillance study. JAMA (J. Am. Med. Assoc.) 253:1749-1754.

3. Scheld, W. M., R. G. Dacey, H. R. Winn, J. E. Welsh, J. A. Jane, and M. A. Sande. 1980. Cerebrospinal fluid outflow resistance in rabbits with experimental meningitis: alterations with penicillin and methylprednisolone. J. Clin. Invest. 66:243-253.

4. Zwahlen, A., J. A. Winkelstein, and E. R. Moxon. 1983. Surface determinants of Haemophilus influenzae pathogenicity: comparative virulence of capsular transformants in normal and complementdepleted rats. J. Infect. Dis. 148:385-394.

5. Syrogiannopoulos, G. A., E. J. Hansen, A. L. Erwin, R. S. Munford, J. Rutledge, J. S. Reisch, and G. H. McCracken, Jr. 1988. Haemophilus influenzae type $b$ lipooligosaccharide induces meningeal inflammation. J. Infect. Dis. 157:237-244.

6. Quagliarello, V. J., W. J. Long, and W. M. Scheld. 1986. Morphologic alteration of the blood-brain barrier with experimental meningitis in the rat. Temporal sequence and role of encapsulation. J. Clin. Invest. 77:1084-1095.

7. Luderitz, O., C. Galanos, and E. T. Rietschel. 1982. Endotoxins of gram-negative bacteria. Pharmacol. Ther. 15:383-402.

8. Meyrick, B. O. 1986. Endotoxin-mediated pulmonary endothelial cell injury. Fed. Proc. 45:19-24.

9. Harlen, J. M., L. A. Harker, M. A. Reidy, C. M. Gajdusek, S. M. Schwartz, and G. E. Striker. 1983. Lipopolysaccharide-mediated bovine endothelial cell injury in vitro. Lab. Invest. 48:269-274.

10. Harlan, J. M., L. A. Harker, S. E. Striker, and L. T. Weaver. 1983. Effect of lipopolysaccharide on human endothelial cells in culture. Thromb. Res. 29:15-26.

11. Heflin, A. C., and K. I. Brigham. 1981. Prevention by granulocyte depletion of increased vascular permeability of sheep lung following endotoxemia. J. Clin. Invest. 68:1253-1260.

12. Kopaniak, M. M., and H. Z. Movat. 1983. Kinetics of acute inflammation induced by Escherischia coli in rabbits. II. The effect of hyperimmunization, complement depletion, and depletion of leukocytes. Am. J. Pathol. 110:13-29.

13. Gaynor, E. 1973. The role of granulocytes in endotoxininduced vascular injury. Blood. 41:797-808.

14. Movat, H. Z., M. I. Cybulsky, I. G. Colditz, M. K. W. Chen, and C. A. Dinarello. 1987. Acute inflammation in gram-negative infection: endotoxin, interleukin 1, tumor necrosis factor, and neutrophils. Fed. Proc. 46:97-104.

15. Eckman, P. L., W. M. King, and J. G. Brunson. 1958. Studies on the blood brain barrier. I. Effects produced by a single injection of gram-negative endotoxin on the permeability of the cerebral vessels. Am. J. Pathol. 34:631-639.
16. Clawson, C. C., J. F. Hartman, and R. L. Vernier. 1966. Electron microscopy of the effect of gram-negative endotoxin on the blood-brain barrier. J. Comp. Neurol. 127:183-198.

17. Schmahl, F. W., W. Schlote, U. Reinhard, H. Heckers, K. Huth, B. Schmid, and E. Betz. 1976. Experimental investigations on the role of the central nervous system in shock induced by intravenous and intracisternal injection of endotoxin. Biochemical, physiological and morphological studies. In Current Topics in Critical Care Medicine. W. C. Shomaker and B. M. Tavares, editors. S. A. Karger, Basel. p. $171-178$.

18. Inzana, T. J., W. E. Seifert, Jr., and R. P. Williams. 1985. Composition and antigenic activity of the oligosaccharide moiety of Haemophilus influenzae type b lipooligosaccharide. Infect. Immun. 48:324-330.

19. Hitchcock, P. J., L. Leive, P. H. Makela, E. T. Rietschel, W. Strittmatter, and D. C. Morrison. 1986. Lipopolysaccharide nomenclature-past, present, and future. J. Bacteriol. 166:699-705.

20. Tauber, M. G., U. Borschberg, and M. A. Sande. 1988. Influence of granulocytes on brain edema, intracranial pressure, and cerebrospinal fluid concentrations of lactate and protein in experimental meningitis. J. Infect. Dis. 157:456-464.

21. Westphal, O., and K. Jann. 1965. Bacterial lipopolysaccharides: extraction with phenol-water and further applications of the procedure. Methods Carbohydrate Chem. Vol. 5:83-91.

22. Johnson, K. G., and M. B. Perry. 1976. Improved techniques for the preparation of bacterial lipopolysaccharides. Can. J. Microbiol. 22:29-34.

23. Gulig, P. A., C. C. Patrick, L. Hermanstoufer, G. H. McCracken, Jr., and E. J. Hansen. 1987. Conservation of epitopes in the oligosaccharide portion of the lipooligosaccharide of Haemophilus influenzae type b. Infect. Immun. 55:513-520.

24. Ingraham, J. L., O. Maaloe, and F. C. Neidhardt. 1983. Growth of the Bacterial Cell. Sinaur Associates, Inc., Sunderland, MA. 3.

25. Alvon, V., V. Naveh, M. Gardos, and A. Greenman. 1971. Neurological sequelae of septic meningitis. Isr. J. Med. Sci. 15:512517

26. Ferry, P. C., J. L. Culbertson, J. A. Cooper, A. B. Silton, and S. H. W. Sell. 1982. Sequelae of Haemophilus influenzae meningitis: preliminary report of a long term follow up study. In Haemophilus influenzae: Epiderniology, Immunology, and Prevention of Diseases. S. H. Sell and P. F. Wright, editors. Elsevier Biomedical Publishers, New York. 111-117.

27. Dodge, P. R., H. Davis, R. D. Feigen, S. J. Holmes, S. L. Kaplan, D. P. Jubelirer, B. W. Stechenberg, and S. K. Hirsh. 1984. Prospective evaluation of hearing impairment as a sequelae of acute bacterial meningitis. N. Engl. J. Med. 311:868-874.

28. Lesse, A. J., E. R. Moxon, A. Zwahlen, and W. M. Scheld. 1988. The role of cerebrospinal fluid pleocytosis and Haemophilus influenzae type $b$ capsule on blood brain barrier permeability during experimental meningitis in the rat. J. Clin. Invest. In press.

29. Deich, R. A., and L. C. Hoyer. 1982. Generation and release of DNA-binding vesicles by Haemophilus influenzae during induction and loss of competence. J. Bacteriol. 152:855-864.

30. Munford, R. S., C. L. Hall, and L. Grimm. 1984. Detection of free endotoxin in cerebrospinal fluid by the Limulus lysate test. Infect. Immun. 45:531-533.

31. Munford, R. S., C. L. Hall, J. M. Lipton, and J. M. Dietschy. 1982. Biological activity, lipoprotein-binding behavior, and in vivo disposition of extracted and native forms of Salmonella typhimurium lipopolysaccharides. J. Clin. Invest. 70:877-888.

32. Tauber, M. G., A. M. Shibl, C. J. Hackbarth, J. W. Larrick, and M. A. Sande. 1987. Antibiotic therapy, endotoxin concentration in cerebrospinal fluid, and brain edema in experimental Escherichia col meningitis in rabbits. J. Infect. Dis. 156:456-462.

33. Berman, N. S., S. E. Siegel, R. Nachum, A. Lipsey, and J. Leedom. 1976. Cerebrospinal fluid endotoxin concentrations in gram negative bacterial meningitis. J. Pediatr. 88:553-556. 
34. Verghese, M. W., and R. Snyderman. 1981. Differential antiinflammatory effects of LPS in susceptible and resistant mouse strains. J. Immunol. 127:288-293.

35. Rosenbaum, J. E., K. T. Hartiala, R. O. Webster, E. L. Howes, Jr., and I. M. Goldstein. 1983. Antiinflammatory effects of endotoxin. Inhibition of rabbit polymorphonuclear leukocyte responses to complement (C5)-derived peptides in vivo and in vitro. Am. J. Pathol. 113:291-299.

36. Dahinden, C., C. Galanos, and J. Fehr. 1983. Granulocyte activation by endotoxin. I. Correlation between adherence and other granulocyte functions, and role of endotoxin structure on biologic activity. J. Immunol. 130:857-862.

37. Sone, S., S. Mriguchi, E. Shimizu, F. Ogushi, and E. Tsubura. 1982. In vitro generation of tumoricidal properties in human alveolar macrophages following interaction with endotoxin. Cancer Res. 42:2227-2231.

38. Davis, B. R., I. S. Barsoum, P. W. Ramwell, and H. Yeager, Jr. 1980. Human alveolar macrophages: effects of endotoxin in vitro. Infect. Immun. 30:753-758.

39. Long, J. B., and J. W. Haladay. 1985. Blood-brain barrier: endogenous modulation by adrenal-cortical function. Science (Wash. DC). 227:1580-1583.

40. Schleimer, R. P., and B: K. Rutledge. 1986. Cultured human vascular endothelial cells acquire adhesiveness for neutrophils after stimulation with interleukin 1, endotoxin, and tumor-promoting phorbol esters. J. Immunol. 136:649-654.

41. Kopaniak, M. M., and H. Z. Movat. 1983. Kinetics of acute inflammation induced by Escherichia coli in rabbits. II. The effect of hyperimmunization, complement depletion, and depletion of leukocytes. Am. J. Pathol. 110:13-29.

42. Tuomanen, E., H. Liu, B. Hengstler, O. Zak, and A. Tomasz.
1985. The induction of meningeal inflammation by components of the pneumococcal cell wall. J. Infect. Dis. 151:859-868.

43. Morrison, D. C., and D. M. Jacobs. 1976. Binding of polymixin B to the lipid A portion of bacterial lipopolysaccharides. Immunochemistry. 13:813-818.

44. Cavaillon, J.-M., and N. Haeffner-Cavaillon. 1986. Polymixin B inhibition of LPS-induced interleukin 1 secretion by human monocytes is dependent upon the LPS origin. Mol. Immunol. 23:965-969.

45. Munford, R. S., and C. L. Hall. 1986. Detoxification of bacterial lipopolysaccharides (endotoxins) by a human neutrophil enzyme. Science (Wash. DC). 234:203-205.

46. Pohlman, T. H., R. S. Munford, and J. M. Harlan. 1987. Deacylated lipopolysaccharide inhibits neutrophil adherence to endothelium induced by lipopolysaccharide in vitro. J. Exp. Med. 165:1393-1402.

47. Morrison, D. C., and J. L. Ryan. 1987. Endotoxins and disease mechanisms. Annu. Rev. Med. 38:417-432.

48. Lebbar, S., J. M. Cavaillon, and M. Caruff. 1986. Molecular requirements for interleukin- 1 induction by lipopolysaccharide stimulated human monocytes: involvement of the heptosyl-2-keto-3-deoxyoctulosonate region. Eur. J. Immunol. 16:87-91.

49. Issekutz, A. C., and S. Bhimji. 1982. Role for endotoxin in the leukocyte infiltration accompanying Escherichia coli inflammation. Infect. Immun. 36:558-566.

50. Dinarello, C. A. 1984. Interleukin-1. Rev. Infect. Dis. 6:51-95.

51. Beutler, B., and A. Cerami. 1987. Cachectin: More than a tumor necrosis factor. N. Engl. J. Med. 316:379-385.

52. Cybulsky, M. I., I. J. Cybulsky, and H. Z. Movat. 1986. The role of interleukin-1 in neutrophil leukocyte emigration induced by endotoxin. Am. J. Pathol. 124:1-9. 